\title{
Targeted DNA damage and repair: the cell's multitool for genome regulation
}

\author{
D.O. Zharkov* \\ Novosibirsk State University, Novosibirsk, Russia \\ Institute of Chemical Biology and Fundamental Medicine SB RAS, Novosibirsk, Russia \\ *e-mail:dzharkov@niboch.nsc.ru
}

Key words: DNA damage, DNA repair, epigenetics, targeted mutagenesis

Motivation and Aim: Cytosine methylation is a generally acknowledged mechanism of gene activity regulation. Disregulation of epigenetic DNA methylation accompanies almost all cancers. While the mechanism of methylation has been known for a while, until recently it was not clear how the 5-methylcytosine $(\mathrm{mC})$ marks could be erased from DNA. In the past few years, it was discovered that $\mathrm{mC}$ is removed by controlled damage by TET1-TET3 dioxygenases and/or AID and APOBEC deaminases followed by excision by DNA glycosylases, the enzymes that so far have been implicated in genome maintenance through DNA repair.

Methods and Algorithms: We have used molecular dynamics computer simulation to explain the specificity of the demethylation enzymes and Monte Carlo simulation to analyze the flows through this pathway. In addition, we have biochemically characterized plant enzymes that directly remove $\mathrm{mC}$ from DNA, and processing of the 5'-adjacent nucleotides during base excision repair.

Results: TET2 dioxygenase and TDG and MBD4 DNA glycosylases, engaged in the active demethylation pathway, seem to derive their specificity for DNA bases in the active demethylation pathway from being able to correctly position them in the active site pocket, unlike the non-substrate bases. The repair steps are limiting the flow through the whole active demethylation pathway. In addition to the canonical pathway direct $\mathrm{mC}$ damage, methylation in human cells can be erased through off-target DNA repair when the initiating lesion resides nearby $\mathrm{mC}$, and artificially erased by foreign $\mathrm{mC}-\mathrm{DNA}$ glycosylases. Overexpression of plant demethylating DNA glycosylase ROS1 in human cells also caused a global decrease in DNA methylation.

Conclusion: Targeted DNA damage and repair emerge as a new paradigm of genome dynamics, in which a number of non-canonical DNA bases are not strictly unwanted lesions that need to be removed as soon as possible but play an important role as regulatory marks.

Acknowledgements: Supported by RSF (No. 17-14-01190), FASO (No. 0309-20180021) and NSU (No. 5-100 Program). 